\title{
Distribution of Staphylococcus haemolyticus as the most dominant species among Staphylococcal infections at the Zainoel Abidin Hospital in Aceh, Indonesia
}

\author{
SUHARTONO SUHARTONO ${ }^{1, \boldsymbol{\nu}}$, ZINATUL HAYATI ${ }^{2}$, MAHMUDA MAHMUDA ${ }^{\mathbf{1}}$ \\ ${ }^{1}$ Department of Biology, Faculty of Mathematics and Natural Sciences, Syiah Kuala University. Jl. Tgk Syech Abdurrauf No.3 Banda Aceh 23111, Aceh, \\ Indonesia. Tel./Fax.+62-651-7410248 •email: suhartono@unsyiah.ac.id. \\ ${ }^{2}$ Department of Microbiology, Faculty of Medicine, Syiah Kuala University, Banda Aceh, Indonesia. Jl. Tgk Tanoh Abee Banda Aceh 23111, Aceh, \\ Indonesia.
}

Manuscript received: 22 June 2019. Revision accepted: 30 June 2019.

\begin{abstract}
Suhartono S, Hayati Z, Mahmuda Mahmuda M. 2019. Distribution of Staphylococcus haemolyticus as the most dominant species among Staphylococcal infections at the Zainoel Abidin Hospital in Aceh, Indonesia. Biodiversitas 20: 2076-2080. The occurrence of Staphylococcus-related infections is emerging and might potentially harbor multidrug resistance leading to the major risks of hospital-associated infection. The study aimed to determine the dominant species distributed among Staphylococcus-related infections from clinical specimens and determine antibiotic resistance profile of the dominant species. The clinical samples were collected from inpatients and outpatients at the Zainoel Abidin Regional Hospital of Aceh, Indonesia during March 2017-March 2018. All clinical samples were mainly inoculated to plates containing blood agar followed by identification and determination of their antibiotic susceptibility using VITEK ${ }^{\circledR} 2$ Compact. Statistical analysis was performed using Chi-square test or Fisher's exact test when appropriate to determine the independence of frequency distributions, and the tests were considered statistically significant at a $P \leq 0.05$ on two-tailed. Of 693 Staphylococcus isolates found in the clinical specimens, Staphylococcus haemolyticus was the most predominant isolates with a total of $233(32.2 \%)$, and it was identified with a high prevalence of methicillin-resistance (95.96\%) termed as methicillin-resistant Staphylococcus haemolyticus (MRSH). MRSH were found in the blood samples and ICU patients accounting for $64.1 \%$ and $55.61 \%$, respectively. The resistance profile of MRSH isolates exhibited a high level of resistance (more than $85 \%$ ) to a wide range of antibiotics, including beta-lactams, (first to the fourth generation) cephalosporins, fluoroquinolones, carbapenems, and macrolides. This might be an alarming occurrence and require serious action to prevent MRSH dissemination of healthcare infections in the future.
\end{abstract}

Keywords: Species distribution, clinical specimens, Staphylococcus haemolyticus, hospital infection

\section{INTRODUCTION}

Nosocomial infections, especially those which are Staphylococcus-related, remain the major problem generating significant implications in clinical and nonclinical settings. These infections might be caused by the occurrence of opportunistic pathogens from both coagulasepositive (CoPS), i.e., Staphylococcus aureus, and coagulasenegative (CoNS) groups. S. aureus has been widely known to be notorious human pathogen causing infections in communal and nosocomial settings with various severities ranging from local and harmless infection to the systemic implications in affected patients (Shinefield and Ruff 2009). Even though the detections and analyses underestimated of CoNS as the current diagnostics emphasize on Staphylococcus aureus (Czekaj et al. 2015), the occurrence of CoNS has been increasingly considered to cause significant infections due to their ability to secrete toxins and form a highly structured biofilm (Rogers et al. 2009).

Antibiotic resistance is emerging in the hospitalassociated infections due to improper administration of antibiotics, i.e. dosages, types, and duration, leading to multidrug-resistant pathogens, including those causing Staphylococcus-related infections (Shinefield and Ruff 2009). The extensive use of antibiotics provides selective pressure to develop resistant strains which results into the reduction of the therapeutic options to treat the infections as well as increasing health risks, complications and patient mortality rates (Hanberger et al. 2014). Therefore, the surveillance of Staphylococcus-related infections and their antibiotic susceptibility, especially in healthcare settings, become crucial. Thus, the objectives of the study were to determine the species distribution of Staphylococcusrelated infections and determine the most prevalent bacterial pathogen along with its antibiotic resistance profile, including groups of methicillin-resistant and methicillin-sensitive, from clinical specimens at the Zainoel Abidin Regional Hospital in Aceh, Indonesia.

\section{MATERIALS AND METHODS}

This study was generated based on survey and analysis of routine diagnostics on the clinical samples received in the Clinical Microbiology Laboratory of the Zainoel Abidin Regional Hospital in Aceh, Indonesia during March 2017-March 2018. The clinical samples used in this study were blood, urine, sputum, body fluid, pus, endotracheal and swabs collected from inpatients and outpatients.

All clinical samples were inoculated to plates 
containing blood agar (Merck, Germany) except for blood samples which were pre-cultured using BacT/ALERT ${ }^{\circledR}$ 3D (Biomeriux, Lyon, France) according to the manufacturer's instructions prior inoculation to the plates. The plates were then incubated for 24 hours at $37^{\circ} \mathrm{C}$ before they were identified morphologically through Gram staining. Identification, as well as antibiotic resistance profile, were generated using VITEK $^{\circledR} 2$ Compact (Biomeriux, Lyon, France) by following the manufacturer's instruction.

Statistical analysis was performed using Chi-square test or Fisher's exact test when appropriate. All tests were performed using XLStat 2016 (Addinsoft, New York, USA), and the tests were considered statistically significant at a $\mathrm{P} \leq 0.05$ on two-tailed.

\section{RESULTS AND DISCUSSION}

Based on the survey and analysis results, it was found that a total of 693 Staphylococcus isolates was recovered and identified on blood agar constituting 206 (29.7\%) isolates of S. aureus and 487 (71.3\%) isolates of coagulasenegative Staphylococci (CoNS) with the most predominant occurrence of S. haemolyticus accounting for 223 (45.8\%) isolates (Figure 1). Figure 1 also highlights the predominant presence of $S$. haemolyticus within clinical samples, which was even more prevalent than coagulasepositive $S$. aureus. Following up these results, the isolates of $S$. haemolyticus were further analyzed to determine their distributions over clinical specimens as well as their antibiotic susceptibility.

Of 223 isolates $(96.6 \%)$ of $S$. haemolyticus found in this study were categorized into methicillin-resistant $S$. haemolyticus (MRSH), whereas, there were only nine isolates of $S$. haemolyticus grouped as methicillin sensitive (MSSH). In terms of sources of clinical samples and the patient care units, there was a difference in distribution between MRSH and MSSH. MRSH was prevalently found in all clinical samples in this study (primarily in the blood samples amounting to $64.1 \%$ ), whereas, MSSH were found in blood, body fluid, and swab samples only (Figure 2).

Regarding the patient care units, both MRSH and MSSH were found in all units of Zainoel Abidin Hospital in Aceh, Indonesia. MRSH was predominantly found in the patients administered in the intensive care unit (ICU) compared to other units. A total of $119(53.4 \%)$ isolates of $S$. haemolyticus collected from the patients in the ICU were methicillin-resistant (Figure 3). Table 1 shows the resistance profiles of $S$. haemolyticus against 32 different antibiotics. It can be observed from the table that the nearly all MRSH isolates (> $80 \%$ of the total MRSH isolates) were highly resistant to both $\beta$-lactam and non- $\beta$-lactam antibiotics, i.e., cephalosporins, macrolides, fluoroquinolones, and carbapenems; and significantly showed different resistance profiles as compared to MSSH. However, MRSH were uniformly susceptible to linezolid, quinupristindalfopristin, doxycycline, minocycline, tigecycline, and nitrofurantoin. It is also important to note that there were only two $(0.9 \%)$ MRSH isolates harboring resistance to vancomycin.

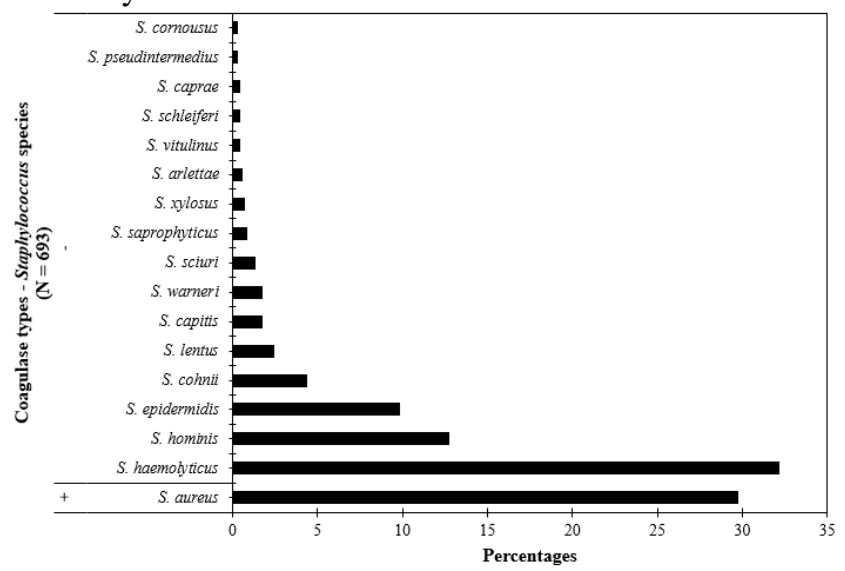

Figure 1. Frequency of occurrences (\%) Staphylococcus-related infections in the clinical specimens from patients at the Zainoel Abidin Regional Hospital in Aceh, Indonesia from March 2017 to March 2018. The number underneath the axis title $(\mathrm{N}=639)$ indicates the total number of isolates recovered from the samples

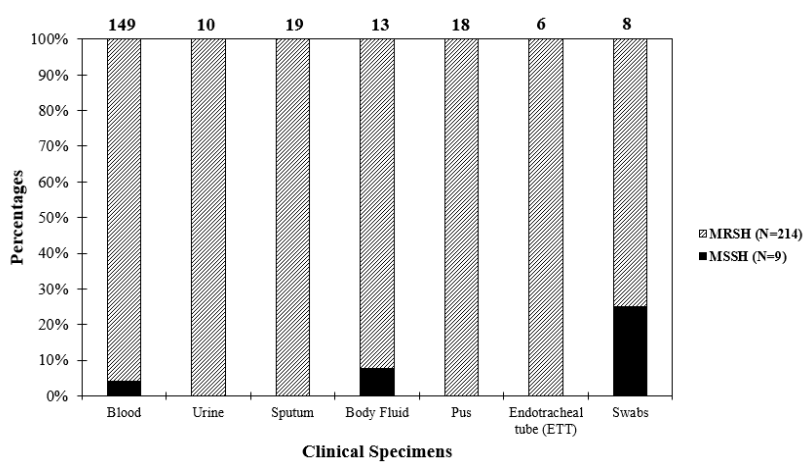

Figure 2. Frequency of occurrence (\%) Methicillin-resistant $S$. haemolyticus (MRSH) $(\mathrm{n}=214)$ and Methicillin-sensitive $S$. haemolyticus (MRSH) $(\mathrm{n}=9)$ across different types of clinical specimens from patients at the Zainoel Abidin Regional Hospital in Aceh, Indonesia during a period of March 2017 - March 2018. Numbers above each column are the total number of isolates recovered from the corresponding clinical samples. Based on the Chi-square test for independence test, the isolates and types of the clinical samples were independent $(P=0.068)$

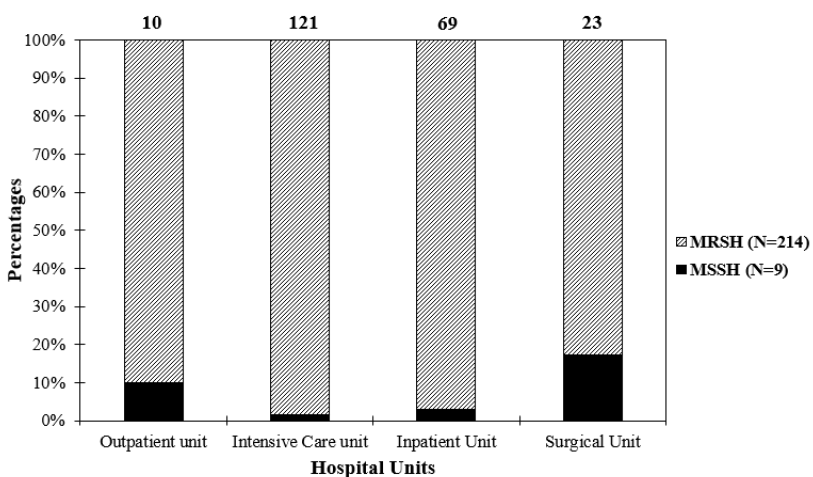

Figure 3. Frequency of occurrence (\%) Methicillin-resistant $S$. haemolyticus (MRSH) $(\mathrm{n}=214)$ and Methicillin-sensitive $S$. haemolyticus (MRSH) $(\mathrm{n}=9)$ from patients at different units of the Zainoel Abidin Regional Hospital in Aceh, Indonesia during a period of March 2017 - March 2018. Numbers above each column are the total number of isolates recovered from the unit. 
Based on the Chi-square test for independence test, the isolates and the patient care units were linked $(P=0.0036)$

Table 1. Resistance profile of methicillin-sensitive S. haemolyticus (MSSH) $(\mathrm{n}=214)$ and methicillin-resistant S. haemolyticus $(\mathrm{MRSH})$ $(\mathrm{n}=9)$ to antibiotics

\begin{tabular}{|c|c|c|c|c|c|c|}
\hline \multirow{2}{*}{ Antibiotics } & \multicolumn{2}{|c|}{ MSSH } & \multicolumn{2}{|c|}{ MRSH } & \multirow{2}{*}{ Chi-2 $^{\mathbf{a}}$} & \multirow{2}{*}{ p-values } \\
\hline & $\mathbf{n}$ & $\%$ & $\mathbf{n}$ & $\%$ & & \\
\hline Amoxicillin & 8 & $(88.9)$ & 214 & $(100.0)$ & 23.885 & $<0.0001$ \\
\hline Amoxicillin/clavulanic acid & 0 & $(0.0)$ & 213 & $(99.5)$ & 199.762 & $<0.0001$ \\
\hline Ampicillin/sulbactam & 0 & $(0.0)$ & 213 & $(99.5)$ & 200.762 & $<0.0002$ \\
\hline Ticarcillin & 8 & $(88.9)$ & 213 & $(99.5)$ & 11.009 & 0.004 \\
\hline Ticarcillin/clavulanic acid & 0 & $(0.0)$ & 212 & $(99.1)$ & 180.749 & $<0.0001$ \\
\hline Piperacillin & 8 & $(88.9)$ & 214 & $(100.0)$ & 23.885 & $<0.0001$ \\
\hline Piperacillin/tazobactam & 0 & $(0.0)$ & 212 & $(99.1)$ & 180.749 & $<0.0001$ \\
\hline Methicillin & 0 & $(0.0)$ & 212 & $(99.1)$ & 181.749 & $<0.0002$ \\
\hline Oxacillin & 0 & $(0.0)$ & 212 & (99.1) & 182.749 & $<0.0003$ \\
\hline Cefoxitin & 0 & $(0,0)$ & 211 & (98.6) & 183.749 & $<0.0004$ \\
\hline Ceftazidime & 0 & $(0,0)$ & 212 & (99.1) & 184.749 & $<0.0005$ \\
\hline Ceftriaxone & 0 & $(0.0)$ & 212 & (99.1) & 185.749 & $<0.0006$ \\
\hline Cefepime & 0 & $(0.0)$ & 212 & $(99,1)$ & 186,749 & $<0.0007$ \\
\hline Imipenem & 0 & $(0.0)$ & 212 & (99.1) & 187,749 & $<0.0008$ \\
\hline Meropenem & 0 & $(0.0)$ & 212 & (99.1) & 188.749 & $<0.0009$ \\
\hline Gentamycin & 0 & $(0.0)$ & 168 & $(78.5)$ & 28,647 & $<0.0001$ \\
\hline Ciprofloxacin & 0 & $(0.0)$ & 194 & (90.7) & 62.739 & $<0.0001$ \\
\hline Mociprofloxacin & 0 & $(0.0)$ & 63 & $(29.4)$ & 3.693 & 0.055 \\
\hline Cefuroxime & 0 & $(0.0)$ & 209 & $(97.7)$ & 140.008 & $<0.0001$ \\
\hline Azithromycin & 1 & (11.1) & 184 & $(86,0)$ & 34.247 & $<0.0001$ \\
\hline Clarithromycin & 1 & (11.1) & 184 & $(86.0)$ & 35.247 & $<0.0002$ \\
\hline Erythromycin & 1 & (11.1) & 184 & $(86.0)$ & 36.247 & $<0.0003$ \\
\hline Clindamycin & 1 & (11.1) & 120 & $(56.1)$ & 7.036 & 0.008 \\
\hline Quinupristin-dalfopristin & 0 & $(0.0)$ & 0 & $(0.0)$ & - & - \\
\hline Linezolid & 0 & $(0.0)$ & 0 & $(0.0)$ & - & - \\
\hline Vancomycin & 0 & $(000)$ & 2 & $(0.9)$ & 0.085 & 0.771 \\
\hline Doxycycline & 0 & $(0.0)$ & 0 & $(0.0)$ & - & - \\
\hline Minocycline & 0 & $(0.0)$ & 0 & $(0,0)$ & - & - \\
\hline Tetracycline & 4 & $(44.4)$ & 49 & $(22.9)$ & 2.213 & 0.137 \\
\hline Tigecycline & 0 & $(0.0)$ & 0 & $(0.0)$ & - & - \\
\hline Nitrofurantoin & 0 & $(0.0)$ & 0 & $(0.0)$ & - & - \\
\hline Trimethoprim/sulfamethoxazole & 0 & $(0.0)$ & 90 & $(42.1)$ & 6.346 & 0.012 \\
\hline Total & 9 & & 214 & & & \\
\hline
\end{tabular}

Note: ${ }^{a}$ Chi-square calculated for comparison of resistance in MSSH versus MRSH. ${ }^{\mathrm{b}} \mathrm{P}$ value generated from the chi-square

\section{Discussion}

Prevalence of hospital-Staphylococcus associated infection, particularly coagulase negative $S$. haemolyticus, is increasing. In this present study, S. haemolyticus isolates were predominantly found (more than 30\%) in different clinical samples of patients in the different units of the hospital. This might signify the characteristics of the opportunistic pathogens which were previously considered as normal flora, commensally found in human skin, particularly in the axilla, perineum, and in inguinal (Takeuchi et al. 2005) similar to their coagulase-positive counterparts S. aureus.

This finding is aligned with previous investigation demonstrating that there were increasing occurrences of coagulase negative-Staphylococcus in blood samples of patients from the hospital in US (Monsen et al. 2005) and Thailand (Teeraputon et al. 2017). Furthermore, coagulasenegative Staphylococcus isolates were also frequently found in samples detected in the blood samples of patients suffering from bacteremia in East Java, Indonesia (Dzen et al. 2013). Together with S. aureus and S. epidermidis, $S$. haemolyticus is recently considered as the three most frequently etiologic agents of Staphylococcus-associated infections (Takeuchi et al. 2005). Despite their lack virulence factors, $S$. haemolyticus strains have the ability to develop multi-resistance to a wide range of antibiotics leading to their clinical significance surpassing the prevalence of other Staphylococcus species (Czekaj et al. 2015).

Among CoNS, S. haemolyticus isolates were isolated second most commonly from patients and healthcare workers suffering from sepsis, peritonitis, otitis, and urinary tract infections generating considerable clinical implications (Correa et al. 2008). This is primarily as a result of their acquirement of the pathogens to develop resistance to antimicrobial agents, including methicillin and/or oxacillin, creating methicillin-resistant $S$. haemolyticus (MRSH). Consequently, these MRSH generate major clinical risks not only by limiting antibiotic options for the treatment, but also transmitting and 
becoming a reservoir of their potential resistance in humans, animals, or both organisms. (Lloyd 2007; Ruzauskas et al. 2014; Sidhu et al. 2007).

In the present study, a total of $214(96 \%)$ isolates were speciated and identified as $S$. haemolyticus with methicillin-resistance determinants due to their low sensitivity to methicillin and/or oxacillin. The high frequency of these MRSH isolates is aligned with previous research suggesting the high prevalence $(77.5 \%)$ of oxacillin-resistant $S$. haemolyticus among coagulasenegative Staphylococcus-related infections (Pereira et al. 2014). Moreover, other studies also reported the high prevalence of MRSH, not only in humans (Teeraputon et al. 2017), but also in animals (Lloyd 2007; Ruzauskas et al. 2014; Sidhu et al. 2007). Additionally, due to their acquired multi-resistance, the MRSH isolates have a crucial role in the transmission of the corresponding resistance genes leading to the widespread of more virulent pathogens, including $S$. aureus, in the clinical settings (Cavanagh et al. 2014).

Based on the types of clinical samples, MRSH isolates were predominantly detected in the blood specimens in the present study. Similar findings were also reported by Teeraputon et al. (2017) The results revealed that out of total isolates $81.9 \%$ of the isolates in the blood samples were $S$. haemolyticus which highlighting the clinical importance of the bacterial isolates in the blood infections (Hitzenbichler et al. 2017). The high prevalence of MRSH isolates within the blood samples might be associated with their pathogenicity since they play a significant role for bacteremia related to colonization during the application of intravascular catheter (Daniel et al. 2014).

In terms of hospital unit in this study, MRSH were more likely to be detected on the clinical specimens of patients who were admitted in the hospital, particularly in the intensive care unit, than outpatients. This result is consistent with a previous study indicating that MRSH is prevalently detected in inpatients accounting for $83.6 \%$ (Ternes et al. 2013). Moreover, MRSH has been identified as the dominant player in catheter-linked bacteremia in the intensive care unit patients (Rodríguez-Aranda et al. 2009) including neonatal and pediatric patients (Hosseinkhani et al. 2018; Ternes et al. 2013). Furthermore, other studies also revealed the high survival and adaptive potential of MRSH strains within healthcare environment as they are detected and cross-transmission not only through medical devices (Kim et al. 2012), but also patients and hospital staff (Squeri et al. 2012).

Of the resistance profile of $\mathrm{MRSH}$ showed that the isolates showed a high level of resistance (more than $85 \%$ ) to a wide range of antibiotics, including beta-lactams, (first to the fourth generation) cephalosporins, fluoroquinolones, carbapenems, and macrolides. This finding corroborates previous investigation signifying the increasing frequency of $S$. haemolyticus strains resistant to the broadest spectrum of antimicrobial agents and disinfectants (Cavanagh et al. 2014; Hope et al. 2008). The development and emerge MRSH resistance to multiple available antibiotics including macrolides, aminoglycosides, quinolones might be linked to the plasticity of the mecA gene, responsible for the expression of modified penicillin-binding (PBP2a) proteins, as well as the presence of insertion sequence (Takeuchi et al. 2005). The MRSH in this study remained sensitive to linezolid, doxycycline, minocycline, tigecycline, nitrofurantoin, and quinupristin-dalfopristin. This result was in parallel with another study elucidating the full susceptibility of MRSH to vancomycin and other drugs of linezolid, daptomycin, tigecycline, quinupristin/dalfopristin (Luiza et al. 2016). However, one thing different from the study of Luiza et al. (2016) is that two $(0.9 \%)$ MRSH isolates in the present study, on the contrary, showed resistance to vancomycin, the last resort of antibiotic from glycopeptides, posing a threat for the persistence and dissemination of the resistance determinants within healthcare settings. In different studies, it was reported the occurrence of MRSH isolates possessed elevating resistance to glycopeptide antibiotics, such as vancomycin (Veach et al. 1990) and teicoplanin (Kristóf et al. 2011) as well as linezolid (Chauhan et al. 2017; Matlani et al. 2016) suggesting the alarming development of MRSH resulting the treatment options to be more limited. Therefore, it is urgently needed to put more attention to the dissemination of the MRSH in the hospital environment through monitoring and surveillance programs so that the risk of infection might be reduced.

In conclusion, of a total of 693 Staphylococcus isolates detected, Staphylococcus haemolyticus had high prevalence, which was dominantly methicillin resistant (MRSH). Predominantly, the MRSH were found in the blood samples of ICU patients, and they were highly resistant to a wide spectrum of antibiotics, including $\beta$ lactams and non- $\beta$-lactams. The MRSH isolates in the present study, however, remained susceptible to linezolid, doxycycline, minocycline, tigecycline, nitrofurantoin, and quinupristin-dalfopristin. Two isolates of $\mathrm{MRSH}$ were noted to be resistant to vancomycin that might pose a threat and might be a crucial factor in the dissemination of healthcare infections.

\section{ACKNOWLEDGEMENTS}

This work was facilitated by the Clinical Microbiology Laboratory of Zainoel Abidin Regional Hospital, Aceh, Indonesia.

\section{REFERENCES}

Cavanagh JP, Hjerde E, Holden MTG, Kahlke T, Klingenberg C, Flægstad T, et al. 2014. Whole-genome sequencing reveals clonal expansion of multiresistant Staphylococcus haemolyticus in European hospitals. J Antimicrob Chemother 69 (11): 2920-2927.

Chauhan D, Verma S, Verma R, Sharma G. 2017. Emergence of resistance to linezolid in methicillin-resistant Staphylococcus haemolyticus reported from the sub-Himalayan region of India. Int $\mathbf{J}$ Res Med Sci 5 (12): 5453-5455.

Correa JE, De Paulis A, Predari S, Sordelli DO, Jeric PE. 2008. First report of qacG, qacH and qacJ genes in Staphylococcus haemolyticus human clinical isolates. J Antimicrob Chemother 62 (5): 956-960.

Czekaj T, Ciszewski M, Szewczyk EM. 2015. Staphylococcus haemolyticus - an emerging threat in the twilight of the antibiotics age. Microbiol 161 (11): 2061-2068. 
Daniel B, Saleem M, Naseer G, Fida AJJoPMS. 2014. Significance of Staphylococcus haemolyticus in hospital-acquired infections. J Pioneer Med Sci 4 (3): 119-125.

Dzen SM, Santoso S, Roekistiningsih R, Santosaningsih D. 2013. Perbedaan pola reistensi Staphylococcus koagulase negative isolate darah terhadap antibiotika di RSU Dr. Saiful Anwar Malang tahun 2000-2001 dengan 2004-2005. J Ked Hewan 21 (3): 127-132.

Hanberger H, Antonelli M, Holmbom M, Lipman J, Pickkers P, Leone M, Rello J, Sakr Y, Walther SM, Vanhems P. 2014. Infections, antibiotic treatment and mortality in patients admitted to ICUs in countries considered to have high levels of antibiotic resistance compared to those with low levels. BMC Infect Dis 14 (1): 513

Hitzenbichler F, Simon M, Salzberger B, Hanses FJI. 2017. Clinical significance of coagulase-negative Staphylococci other than $S$ epidermidis bloodstream isolates at a tertiary care hospital. Infection 45 (2): 179-186.

Hope R, Livermore DM, Brick G, Lillie M, Reynolds R, on behalf of the BWPoRS. 2008. Non-susceptibility trends among Staphylococci from bacteraemias in the UK and Ireland, 2001-06. J Antimicrob Chemother 62: ii65-ii74.

Hosseinkhani F, Tammes Buirs M, Jabalameli F, Emaneini M, van Leeuwen WB. 2018. High diversity in SCCmec elements among multidrug-resistant Staphylococcus haemolyticus strains originating from paediatric patients; characterization of a new composite island. J Med Microbiol 67 (7): 915-921.

Kim JS, Kim HS, Park JY, Koo HS, Choi CS, Song W, et al. 2012. Contamination of X-ray cassettes with methicillin-resistan Staphylococcus aureus and methicillin-resistant Staphylococcus haemolyticus in a Radiology Department. Ann Lab Med 32 (3): 206209.

Kristóf K, Kocsis E, Szabó D, Kardos S, Cser V, Nagy K, et al. 2007. Significance of methicillin-teicoplanin resistant Staphylococcus haemolyticus in bloodstream infections in patients of the Semmelweis University hospitals in Hungary. Eur J Clin Microbiol Infect Dis 2011;30 (5): 691-9.

Lloyd DH. Reservoirs of antimicrobial resistance in pet animals. Clin Infect Dis 45: S148-S52.

Luiza P, Ivo BC, Cataneli PV, Adilson O, Rocha BA, Henrique CC, et al. 2016. Susceptibility profile of Staphylococcus epidermidis and Staphylococcus haemolyticus isolated from blood cultures to vancomycin and novel antimicrobial drugs over a period of 12 Years. Microb Drug Resist 22 (4): 283-93.

Matlani M, Shende T, Bhandari V, Dawar R, Sardana R, Gaind R. 2016. Linezolid-resistant mucoid Staphylococcus haemolyticus from a tertiary-care centre in Delhi. New Microbes New Infect 11: 57-58.
Monsen T, Karlsson C, Wistrom J. 2005. Spread of clones of multidrugresistant, coagulase negative Staphylococci within a university hospital. Infect Control Hosp Epidemiol 26 (1): 76-80.

Pereira PMA, Binatti VB, Sued BPR, Ramos JN, Peixoto RS, Simões C, et al. 2014. Staphylococcus haemolyticus disseminated among neonates with bacteremia in a neonatal intensive care unit in Rio de Janeiro, Brazil. Diagn Microbiol Infect Dis 78 (1): 85-92.

Rodríguez-Aranda A, Daskalaki M, Villar J, Sanz F, Otero JR, Chaves F. Nosocomial spread of linezolid-resistant Staphylococcus haemolyticus infections in an intensive care unit. Diagn Microbiol Infect Dis 63 (4): 398-402.

Rogers KL, Fey PD, Rupp ME. 2009. Coagulase-negative Staphylococcal infections. Infect Dis Clin North Amer 23 (1): 73-98.

Ruzauskas M, Siugzdiniene R, Klimiene I, Virgailis M, Mockeliunas R, Vaskeviciute L, et al. 2014. Prevalence of methicillin-resistant Staphylococcus haemolyticus in companion animals: a cross-sectional study. Ann Clin Microbiol Antimicrob 13 (1): 56.

Shinefield HR, Ruff NL. 2009. Staphylococcal infections: A historical perspective. Infect Dis Clin North Amer 23 (1): 1-5.

Sidhu MS, Oppegaard H, Devor TP, Sørum H. 2007. Persistence of multidrug-resistant Staphylococcus haemolyticus in an animal veterinary teaching hospital clinic. Microb Drug Resist 13 (4): 271280.

Squeri R, Grillo OC, La Fauci V. 2012. Surveillance and evidence of contamination in hospital environment from meticillin and vancomycin-resistant microbial agents. J Prev Med Hyg 53 (3): 143145.

Takeuchi F, Watanabe S, Baba T, Yuzawa H, Ito T, Morimoto Y, et al 2005. Whole-genome sequencing of Staphylococcus haemolyticus uncovers the extreme plasticity of its genome and the evolution of human-colonizing Staphylococcal Species. J Bacteriol 187 (21): 7292-7308.

Teeraputon S, Santanirand P, Wongchai T, Songjang W, Lapsomthob N, Jaikrasun D, et al. 2017. Prevalence of methicillin resistance and macrolide-lincosamide-streptogramin B resistance in Staphylococcus haemolyticus among clinical strains at a tertiary-care hospital in Thailand. New Microbes New Infect 19: 28-33.

Ternes YM, Lamaro-Cardoso J, André MCP, Pessoa VP, Vieira MAdS, Minamisava R, et al. 2013. Molecular epidemiology of coagulasenegative Staphylococcus carriage in neonates admitted to an intensive care unit in Brazil. BMC Infect Dis 13: 572. DOI: 10.1186/14712334-13-572.

Veach LA, Pfaller MA, Barrett M, Koontz FP, Wenzel RP. 1990. Vancomycin resistance in Staphylococcus haemolyticus causing colonization and bloodstream infection. J Clin Microbiol 28 (9): 2064-2068 